\title{
Vaginal Cuff Dehiscence After Robotic Total Laparoscopic Hysterectomy
}

\author{
Barbara L. Robinson, MD, John B. Liao, MD, PhD, Sarah F. Adams, MD, and Thomas C. \\ Randall, MD \\ Department of Obstetrics and Gynecology, Pennsylvania Hospital, 800 Spruce Street, Philadelphia, \\ PA 19107, Division of Gynecologic Oncology, Department of Obstetrics and Gynecology, University \\ of Pennsylvania, 3400 Civic Center Boulevard, Philadelphia, PA 19104
}

\begin{abstract}
BACKGROUND—Vaginal cuff dehiscence with small bowel evisceration after hysterectomy is a rare event that may be occurring more frequently with the advent of robotic laparoscopic hysterectomies.

CASES-Two women underwent robotic total laparoscopic hysterectomy for menorrhagia and stage I endocervical adenocarcinoma, respectively. Each presented 7-8 weeks postoperatively with abdominal pain and vaginal pressure after intercourse. The small bowel protruded into the vagina through the dehisced vaginal cuff. Both cuffs were repaired vaginally with delayed absorbable suture. One repair required revision 7 weeks after the initial repair.

CONCLUSION-Robotic total laparoscopic hysterectomy may be associated with increased risk of vaginal cuff dehiscence and small bowel evisceration. This observation may be because of thermal spread and cuff tissue damage from electrosurgery used for colpotomy.
\end{abstract}

Vaginal cuff dehiscence and subsequent small bowel evisceration after hysterectomy is a rare event with only 59 cases reported between January 1900 and December 2001. ${ }^{1}$ Ninety-five percent of cases occurred after total abdominal or total vaginal hysterectomy (TAH, TVH). Recent reviews describe a disproportionate number of vaginal cuff dehiscences occurring after total laparoscopic hysterectomy (TLH), suggesting that vaginal cuff dehiscence may be a more common complication of laparoscopic hysterectomy than TAH or TVH. ${ }^{2,3}$ We describe two cases of vaginal cuff dehiscence and small bowel evisceration after robotic TLH.

\section{CASE 1}

A 48-year-old woman with a history of left breast ductal carcinoma in situ, recurrent bilateral ovarian cysts, and heavy menses underwent a robotic TLH for treatment of menorrhagia and bilateral salpingo-oophorectomy for cancer risk reduction. She had no prior abdominal or pelvic surgeries. Total operative time was 3 hours. Estimated blood loss was $50 \mathrm{~mL}$, and there were no complications. The patient was discharged home on postoperative day 1. Eight weeks (59 days) postoperatively, the patient presented shortly after intercourse complaining of dyspareunia, abdominal cramping, bloody vaginal discharge, and the sensation of something coming from the vagina with Valsalva maneuver. She had had two prior episodes of intercourse without event. On examination, loops of small bowel were protruding through the vaginal cuff.

Corresponding author: Thomas C. Randall, MD, Department of Obstetrics and Gynecology, University of Pennsylvania, 801 Spruce Street, 7th Floor, Philadelphia, PA 19107; Thomas.Randall@uphs.upenn.edu.

Financial Disclosure

The authors did not report any potential conflicts of interest. 
The eviscerated bowel was minimally inflamed and not densely adherent to the edges of the vaginal cuff. The vaginal cuff edges appeared pink and healthy but were not bleeding. Urgent transvaginal cuff closure was performed using braided delayed absorbable suture in a running, non-locking fashion. Operative time was 15 minutes, and estimated blood loss was less than $25 \mathrm{~mL}$. The patient was discharged home on postoperative day 1 . On postoperative day 51 (7 weeks) after transvaginal cuff closure, the patient was seen for a postoperative examination. The vaginal cuff opened with gentle digital pressure. In the operating room, the cuff edges were revised and reapproximated in a transverse fashion using interrupted, monofilament delayed absorbable sutures. The patient was discharged home that day.

\section{CASE 2}

A 57-year-old woman underwent a robotic modified radical hysterectomy, bilateral salpingooophorectomy, and pelvic and paraaortic lymph node dissection for endocervical adenocarcinoma. The only prior pelvic or abdominal surgery included a cesarean delivery at 28 weeks of gestation for severe preeclampsia, which was complicated by a wound dehiscence requiring surgical repair. Operative time was 4 hours, and estimated blood loss was $50 \mathrm{~mL}$. The patient was discharged on postoperative day 2 . Seven weeks (52 days) later, the patient presented with diffuse abdominal and rectal pain during intercourse. The pain resolved, but she felt pressure in the vagina with Valsalva. On examination, a loop of small bowel was seen in the vagina. The cuff appeared granulated but hemostatic, and there was no evidence of residual suture, incarcerated intestine, or peritoneal inflammation. Approximately $5 \mathrm{~mm}$ of the cuff edge was resected to expose fresh vaginal tissue. The revised vaginal cuff edges were reapproximated with interrupted braided absorbable suture. The patient was discharged home on postoperative day 1 .

Both hysterectomies were completed using the da Vinci Surgical System (Intuitive Surgical, Sunnydale, CA). After isolation, ligation, and transection of the infundibulopelvic ligament, uterine vessels, round ligaments, and cardinal and uterosacral ligaments, a circumferential colpotomy was made using monopolar scissors on coagulation mode at 50 watts. The specimen was then placed in an Endo Catch bag (Covidien's Autosuture, Mansfield, MA) and evacuated through the colpotomy, which was then reapproximated in a running, nonlocking fashion using number 0 braided absorbable suture on a GS 23 needle. Care was taken to include the peritoneum and uterosacral ligaments in the closure. Sutures were placed beyond the visibly desiccated cuff edges to include visibly viable tissue. Knots were tied intracorporeally. Prophylactic antibiotics were administered before all procedures. The postoperative courses for both patients, in the hospital and as outpatients, were uncomplicated except as noted previously. Additionally, there were no signs and symptoms of infection as both patients remained afebrile without leukocytosis.

\section{COMMENT}

In April 2005, the da Vinci Surgical System (Intuitive Surgical, Sunnydale, CA) was approved by the FDA to perform gynecologic laparoscopic procedures. In some instances, robotic hysterectomy may become the preferred procedure over laparoscopic and abdominal hysterectomy as it may be associated with reduced blood loss and length of hospital stay. ${ }^{4}$ Thus, any possibly associated complications, such as vaginal cuff dehiscence, should be investigated. While the overall incidence of vaginal cuff dehiscence and subsequent small bowel evisceration is unknown, recent literature suggests that this complication is becoming more common and may be associated with increasing numbers of laparoscopic and robotic hysterectomies. A single institution observational case series that included 7,039 total hysterectomies between January 2000 and March 2006 reported 10 vaginal cuff dehiscences with an overall incidence of $0.14 \%$. In this series, the majority of the cases occurred during the 
last 15 months of the study period with a cumulative incidence of vaginal cuff dehiscence of $4.93 \%$ among TLHs, $0.29 \%$ among TVHs, and $0.12 \%$ among TAHs. ${ }^{2}$ Another study reported three cases of vaginal cuff dehiscence occurring shortly after intercourse or straining to have a bowel movement within 2-5 months after TLH for benign indications. ${ }^{3}$ A retrospective review of 2,399 hysterectomies performed at the Mayo Clinic in Scottsdale, Arizona, of which $15 \%$ were performed robotically and $9 \%$ with traditional laparoscopy, reported the vaginal cuff dehiscence rate to be $2.87 \%$ for robotic hysterectomy and $0.47 \%, 0.13 \%$, and $0.99 \%$ for TLH, $\mathrm{TVH}$, and TAH, respectively. ${ }^{5}$ At our institution, there have been two cases of vaginal cuff dehiscence among the 205 robotic TLHs performed between December 2007 and January 2009 , with an incidence of $0.98 \%$.

Colpotomy technique may account for the observed increased risk of vaginal cuff dehiscence associated with robotic and traditional TLH as compared with TVH and TAH. In the latter procedures, the colpotomy is often made sharply using heavy scissors or a knife. However, during robotic and traditional TLH, the colpotomy is often made using electrosurgery, which may yield thermal damage at the cuff site, weakening the tissue and increasing the risk of dehiscence. At our institution, we have traditionally used monopolar scissors in the coagulation mode for the colpotomy. Coagulation mode is an interrupted, high-voltage current dispersed over a large surface area, while cutting mode is a continuous, low-voltage current concentrating the energy in a small area, resulting in more rapid tissue heating and less thermal spread. Coagulation mode may result in more tissue damage, but is better at sealing vessels in vascular areas like the vaginal cuff. In our institution, we now use the monopolar hook and a blend of coagulation and cutting modes to theoretically reduce the risk of vaginal cuff dehiscence. Alternatively, a harmonic scalpel which uses a high frequency vibrating blade for cutting may be used during both robotic and traditional TLH. Finally, the use of interrupted sutures rather than a single continuous suture for cuff closure may help avoid complete dehiscence.

Ultimately, the cause for these dehiscences is unknown, and multiple factors may be involved. Prior studies have suggested several other factors that may increase risk of vaginal cuff dehiscence and small bowel evisceration, including sexual intercourse, increased intraabdominal pressure from frequent straining or Valsalva, vaginal trauma, smoking, menopause, history of pelvic surgery, connective tissue disease, prior vaginoplasty, and corticosteroid use. ${ }^{6,7}$ Additionally, while there is no consensus on the proper management of this gynecologic emergency, most agree that urgent surgical repair with aggressive intravenous fluid resuscitation and prophylactic antibiotics are essential. Vaginal repair of the dehiscence is appropriate unless the bowel cannot be easily reduced through the vagina or there are features of peritonitis or impaired bowel viability. ${ }^{8}$

In conclusion, vaginal cuff dehiscence is a rare gynecologic emergency that may occur more frequently with robotic total laparoscopic hysterectomy. More studies are needed to confirm this observation and identify an etiology.

\section{References}

1. Ramirez PT, Klemer DP. Vaginal evisceration after hysterectomy: a literature review. Obstet Gynecol Surv 2002;57:462-7. [PubMed: 12172223]

2. Hur HC, Guido RS, Mansuria SM, Hacker MR, Sanfilippo JS, Lee TT. Incidence and patient characteristics of vaginal cuff dehiscence after different modes of hysterectomies. J Minim Invasive Gynecol 2007;14:311-7. [PubMed: 17478361]

3. Nezhat CH, Nezhat F, Seidman DS, Nezhat C. Vaginal vault evisceration after total laparoscopic hysterectomy. Obstet Gynecol 1996;87:868-70. [PubMed: 8677119]

4. Magrina JF, Kho RM, Weaver AL, Montero RP, Magtibay PM. Robotic radical hysterectomy: comparison with laparoscopy and laparotomy. Gynecol Oncol 2008;109:86-91. [PubMed: 18279944] 
5. Akl, MN. Robotic hysterectomy more likely to result in vaginal cuff dehiscence. Annual Meeting of the Society of Gynecologic Surgeons; 2008.

6. Croak AJ, Gebhart JB, Klingele CJ, Schroeder G, Lee RA, Podratz KC. Characteristics of patients with vaginal rupture and evisceration. Obstet Gynecol 2004;103:572-6. [PubMed: 14990423]

7. Nasr AO, Tormey S, Aziz MA, Lane B. Vaginal herniation: case report and review of the literature. Am J Obstet Gynecol 2005;193:95-7. [PubMed: 16021065]

8. Kim SM, Choi HS, Byun JS, Kim YS, Kim HR. Transvaginal evisceration after radical abdominal hysterectomy. Gynecol Oncol 2002;85:543-4. [PubMed: 12051889] 